\title{
The Transformation of a Schwarzschild Black Hole Linear Perturbations to Bondi Frame
}

\author{
Amos S. Kubeka1, Nigel T. Bishop ${ }^{2}$ \\ ${ }^{1}$ Department of Mathematical Sciences, University of South Africa, Unisa, South Africa \\ ${ }^{2}$ Department of Mathematics (Pure and Applied), Rhodes University, Grahamstown, South Africa \\ Email: kubekas@unisa.ac.za, n.bishop@ru.ac.za
}

Received 12 August 2014; revised 8 September 2014; accepted 1 October 2014

Copyright (C) 2014 by authors and Scientific Research Publishing Inc.

This work is licensed under the Creative Commons Attribution International License (CC BY). http://creativecommons.org/licenses/by/4.0/

c) (i) Open Access

\section{Abstract \\ We extend standard linear perturbations of a Schwarzschild black hole by Chandrasekhar to Bon- di frame by transforming both even and odd parity perturbations when angular momentum $I=2$.}

\section{Keywords}

Black Hole Linear Perturbations, Null Cone Formalism, Bondi-Sachs Formalism, Odd Parity Perturbations, Even Parity Perturbations

\section{Introduction}

In studying linear perturbations of a Schwarzschild black hole we are able to study its static space-time properties and the emission of gravitation radiation. The gravitational radiation emitted by a Schwarzschild black hole carries information about its mass (as well as spin and charge for rotating and/or charged black holes). Also by studying the perturbations of a Schwarzschild black hole it is possible to make conclusions about the stability of the Einstein equations [1]. Because of the challenges of studying the gravitational radiation analytically, people have developed numerical techniques [2] to solve the field equations by evolving the metric. Different approaches are used in numerical relativity to tackle these problems in standard coordinates, the most approach being the ADM formalism [3] [4] which is based on the split of spacetime into space and time. However, the natural formalism based on the fact that gravitational radiation travels at the speed of light and uses null coordinates, is called Bondi-Sachs formalism [5] [6]. Important numerical studies involving black hole-black hole, black hole-neutron star, and neutron star-neutron star binaries have been done [7]-[12] in this direction.

In this paper we transform well-known linear perturbations of a Schwarzschild black hole to Bondi-Sachs 
form. The outline of this paper is as follows: in Section 2 we discuss the Bondi-Sachs formalism as background material. In Section 3, we discuss linearized Bondi-Sachs metric. In Section 4, we discuss the complex notation to be used. In Section 5, we transform the linear perturbations of a Schwarzschild black hole to Bondi-Sachs frame. Section 6 is a discussion. The paper ends with the conclusion in Section 7.

\section{Background Material}

We use coordinates based upon a family of outgoing null hypersurfaces $u=c o n t$, where $u$ is the retarded time parameter. We let $x^{A} \quad(A=2,3)$ be the null rays, and $r$ be a surface area coordinate. In the resulting $x^{i}=\left(u, r, x^{A}\right)$ coordinates, the metric takes the BS form [5] [13]

$$
\begin{aligned}
\mathrm{d} s^{2}= & -\left[\mathrm{e}^{2 \beta}\left(1+\frac{W}{r}\right)-r^{2} h_{A B} U^{A} U^{B}\right] \mathrm{d} u^{2}-2 \mathrm{e}^{2 \beta} \mathrm{d} u \mathrm{~d} r \\
& -2 r^{2} h_{A B} U^{B} \mathrm{~d} u \mathrm{~d} x^{A}+r^{2} h_{A B} \mathrm{~d} x^{A} \mathrm{~d} x^{B},
\end{aligned}
$$

where $h^{A B} h_{B C}=\delta_{B}^{A}$ and $\operatorname{det}\left(h_{A B}\right)=\operatorname{det}\left(q_{A B}\right)$, with $q_{A B}$ being a unit sphere metric.

We work in spherical polar coordinates $x^{A}=(\theta, \phi)$ and the unit sphere metric is given by

$$
\begin{aligned}
q_{A B} \mathrm{~d} x^{A} \mathrm{~d} x^{B} & =g_{22} \mathrm{~d} x^{2} \mathrm{~d} x^{2}+g_{23} \mathrm{~d} x^{2} \mathrm{~d} x^{3}+g_{32} \mathrm{~d} x^{3} \mathrm{~d} x^{2}+g_{33} \mathrm{~d} x^{3} \mathrm{~d} x^{3} \\
& =g_{22} \mathrm{~d} \theta^{2}+\left(g_{23}+g_{32}\right) \mathrm{d} \theta \mathrm{d} \phi+g_{33} \mathrm{~d} \phi^{2} .
\end{aligned}
$$

We now introduce the complex dyad $q_{A}(A=\theta, \phi)=(1, i \sin \theta)$ where $q^{A}=\left(1, \frac{j}{\sin \theta}\right)$ and $j=\sqrt{-1} \cdot q_{A}$ and $q^{A}$ satisfy the following conditions: $q^{A} q_{A}=0, q^{A} \bar{q}_{A}=2$, and $q^{A}=q^{A B} q_{B}$, with $q^{A B} q_{B C}=\delta_{C}^{A}$ and $q_{A B}=\frac{1}{2}\left(q_{A} \bar{q}_{B}+\bar{q}_{A} q_{B}\right)$, where $\bar{q}_{A}$ and $\bar{q}_{B}$ are the complex dyad conjugate of $q_{A}$ and $q_{B}$ respectively.

We also introduce the complex quantities $U, J$ defined by

$$
U=q_{A} U^{A},
$$

and

$$
J=q^{A} q^{B} h_{A B} / 2 .
$$

For spherically symmetric case (Schwarzschild space-time), we take $J=0$ and $U=0$. $J$ and $U$ are interlinked, and they contain all the dynamic content of the gravitational filed in the linearized regime [14]. Lastly we introduce the complex differential eth operators $\bar{\partial}$ and $\bar{\partial}$ (see [15] for full details). The eth ( $\check{\partial}$ ) formalism gives a compact and efficient manner of treating vector and tensor fields on the sphere, as well as their covariant derivatives.

We define the operator $\partial$ acting on a quantity $\mathcal{V}$ of spin-weight $s$, as

$$
\widetilde{\mathcal{V}}=-(\sin \theta)^{s}\left[\frac{\partial}{\partial \theta}+j \csc \theta \frac{\partial}{\partial \phi}\right](\sin \theta)^{-s} \mathcal{V}
$$

which has the property of raising the spin-weight by 1 , and similarly we define $\bar{\delta}$ as

$$
\bar{\partial} \mathcal{V}=-(\sin \theta)^{-s}\left[\frac{\partial}{\partial \theta}-j \csc \theta \frac{\partial}{\partial \phi}\right](\sin \theta)^{s} \mathcal{V}
$$

which has the property of lowering the spin-weight by 1 .

For a Schwarzschild space-time, we have $J=U=0$, and usually we can describe this space-time by $\beta=0$ and $W=-2 M$, or by $\beta=\beta_{c}$ (constant) and $W=\left(\mathrm{e}^{2 \beta_{c}}-1\right) r-2 M$. For a spherically symmetric spacetime, $J$ and $U$ are zero and thus they can be regarded as a measure of the deviation from spherical symmetry, and in addition, they carry the gravitational radiation information.

\section{Linearized Bondi-Sachs Metric}

We linearize Bondi-Sachs metric in order to find $J, U, \beta$, and $w$ in the next section from the transformed linear 
perturbations of a Schwarzschild black hole in the case $l=2$. Bondi-Sachs metric linearized about Schwarzschild background has the following metric components

$$
\begin{aligned}
& g_{00}=-1+\frac{2 M}{r}-2 \beta+4 M \beta-\frac{w}{r} \\
& g_{01}=g_{10}=-1-2 \beta \\
& g_{02}=g_{20}=-r^{2} U^{\theta} \\
& g_{03}=g_{30}=-r^{2} \sin ^{2} \theta U^{\phi} \\
& g_{11}=g_{12}=g_{13}=g_{21}=g_{31}=0 \\
& g_{23}=g_{32}=r^{2} b=r^{2} h_{23}=r^{2} h_{32} \\
& g_{22}=r^{2}(1+a)=r^{2} h_{22} \\
& g_{33}=r^{2}(1-a) \sin ^{2} \theta=r^{2} h_{33}
\end{aligned}
$$

where $a$ and $b$ are functions of $r$ and $\theta$ only, and metric quantities $\beta, w, U^{\theta}$ and $U^{\phi}$ are all small. We write $\beta, U, J$ and $w$ explicitly as:

$$
\beta=\beta_{0} \mathrm{e}^{\mathrm{i} \sigma u} Y_{20}(\theta)
$$

From Equation (3) we have

$$
\begin{aligned}
U & =U^{\theta}+j \frac{U^{\phi}}{\sin \theta}=-\frac{1}{r^{2}}\left[g_{02}+j \frac{g_{03}}{\sin \theta}\right] \\
& =U_{0}(r) \mathrm{e}^{i \sigma u} \sqrt{6}_{1} Y_{20}(\theta) .
\end{aligned}
$$

From Equation (4) we have

$$
J=\frac{1}{r^{2}} \frac{q^{A} q^{B} g_{A B}}{2}=a+j \frac{b}{\sin \theta}=J_{0}(r) \mathrm{e}^{i \sigma u} 2 \sqrt{6}_{2} Y_{20}(\theta) .
$$

Lastly

$$
w=w_{0}(r) \mathrm{e}^{\mathrm{i} \sigma u} Y_{20}(\theta)
$$

The spherical harmonics $Y_{20}(\theta),{ }_{2} Y_{20}(\theta)$, and ${ }_{1} Y_{20}(\theta)$ are respectively given by

$$
\begin{aligned}
& Y_{20}(\theta)=\left(\frac{5^{\frac{1}{2}}}{4 \pi^{\frac{1}{2}}}\right)\left(2-3 \sin ^{2} \theta\right), \\
& { }_{2} Y_{20}(\theta)=\left(\frac{5^{\frac{1}{2}}}{4 \pi^{\frac{1}{2}}}\right) 3(1-\cos 2 \theta), \\
& { }_{1} Y_{20}(\theta)=-\left(\frac{5^{\frac{1}{2}}}{4 \pi^{\frac{1}{2}}}\right) 3 \sin 2 \theta .
\end{aligned}
$$

\section{Complex Notation}

At this stage we must deal with a notational issue concerning the use of complex numbers to represent physical 
quantities. $J$ and $U$ are complex and are used as a convenient representation of metric quantities with two real components. However, it is also common practice to represent oscillations in time as $\mathrm{e}^{i \sigma u}$. More precisely, it is common to write

$$
A_{R} \cos \sigma u-A_{I m} \sin \sigma u=\operatorname{Re}\left\{\left(A_{R}+i A_{I m}\right) \mathrm{e}^{\mathrm{i} \sigma u}\right\}=\operatorname{Re}\left\{A \mathrm{e}^{\mathrm{i} \sigma u}\right\}
$$

with $A=A_{R}+i A_{\text {Im }}$.

Not only is the above a more compact notation, but also it is much easier to manipulate $\mathrm{e}^{\mathrm{i} \sigma u}$ (by means of differential and integral operators) than sine or cosine function.

The difficulty is that the complex nature of $J$ and $U$ on the one hand, and of $\mathrm{e}^{\mathrm{i} \sigma u}$ on the other, have no connection with each other. The simplest way around the problem is to keep complex representations for both $\mathrm{e}^{\mathrm{i} \sigma u}$, as well as $J$ and $U$, by using $i$ in $\mathrm{e}^{i \sigma u}$ with $i^{2}=-1$, and $j$ in $J$ and $U$ with $j^{2}=-1$, but $i \neq j$ and $i j$ not simplifiable. Although this construction appears similar to quaternion theory, it is, in fact, different. A new algebra has not been constructed, and only addition and multiplication will be performed. In general, an inverse may not exist, so division is not permitted.

The above construction was not made in [16] because in that work it was possible to neglect the imaginary component in $J$ and $U$. However, we shall see that it is not the case for odd-parity perturbations.

\section{Transformation of Linear Perturbations to Bondi-Sachs form}

The general metric for time-dependent axisymmetric systems in general coordinates $\left(t, x^{2}, x^{3}, \phi\right)$ is given by [17]

$$
\mathrm{d} s^{2}=\mathrm{e}^{2 v}(\mathrm{~d} t)^{2}-\mathrm{e}^{2 \lambda}\left(\mathrm{d} \phi-\omega \mathrm{d} t-q_{2} \mathrm{~d} x^{2}-q_{3} \mathrm{~d} x^{3}\right)^{2}-\mathrm{e}^{2 \mu_{2}}\left(\mathrm{~d} x^{2}\right)^{2}-\mathrm{e}^{2 \mu_{3}}\left(\mathrm{~d} x^{3}\right)^{2},
$$

where $v, \psi, \mu_{2}, \mu_{3}, \omega, q_{2}$, and $q_{3}$ are functions of time $t$, and $x^{2}$, and $x^{3}$ are independent of $\phi$. The unperturbed Schwarzschild metric which is the special solution to Equation (23) in spherical coordinates $(t, r, \theta, \phi)$ is given by

$$
\mathrm{ds}^{2}=-\left(1-\frac{2 M}{r}\right) \mathrm{d} t^{2}+\left(1-\frac{2 M}{r}\right)^{-1} \mathrm{~d} r^{2}+r^{2}\left(\mathrm{~d} \theta^{2}+\sin ^{2} \theta \mathrm{d} \phi^{2}\right) .
$$

where $x^{2}=r, x^{3}=\theta$, and $\mu_{2}=\lambda$ (say), $\mathrm{e}^{2 v}=\mathrm{e}^{-2 \lambda}=1-2 m / r, \mathrm{e}^{\psi}=r \sin \theta, \mathrm{e}^{\mu_{3}}=r$, and $\omega=q_{2}=q_{3}=0$.

When the Schwarzschild metric is perturbed we have $v+\delta v, \lambda+\delta \lambda$, and $\mu_{3}+\delta \mu_{3}$ (for even-parity perturbations), and $\omega, q_{2}$, and $q_{3}$ are taken as quantities of the first order of smallness (for odd-parity perturbations) as it is the case with $\delta v, \delta \lambda$, and $\delta \mu_{3}$.

\subsection{Even-Parity Metric Perturbations}

\subsubsection{The Transformation Procedure}

We start by transforming $t$ to $u$ by performing the following transformation

$$
u=t-F(r)-\varepsilon f(r) \mathrm{e}^{i \sigma t} P_{2}(\theta)
$$

where $F(r)$ and $f(r)$ are functions that needs to be determined and $P_{2}(\theta)=\left[\frac{3}{2} \cos ^{2} \theta-\frac{1}{2}\right]$ are the Legendre functions. Differentiating Equation (25) we get

$$
\begin{aligned}
\mathrm{d} t= & \mathrm{d} u+\varepsilon f(r) i \sigma \mathrm{e}^{i \sigma t} P_{2}(\theta) \mathrm{d} u+F(r)_{, r} \mathrm{~d} r+\varepsilon F(r)_{, r} f(r) i \sigma \mathrm{e}^{i \sigma t} P_{2}(\theta) \mathrm{d} r \\
& +\varepsilon f(r)_{, r} \mathrm{e}^{i \sigma t} P_{2}(\theta) \mathrm{d} r+\varepsilon f(r) \mathrm{e}^{i \sigma t} P_{2}(\theta)_{, \theta} \mathrm{d} \theta .
\end{aligned}
$$

Then we substitute Equation (26) into the perturbed metric and we chose a function $F(r)$ such that the transformed metric after the substitution of Equation (26) has the coefficient of $\mathrm{dr}^{2}$ zero to the zeroth order in $\varepsilon$. Similarly we chose a function $f(r)$ such that the coefficient of $\mathrm{d}^{2}$ is zero to 1st order in $\varepsilon$. We found functions $F(r)$ and $f(r)$ to be 


$$
F(r)=r+2 M \ln (r-2 M)
$$

and

$$
f(r)=\mathrm{e}^{\left(\frac{r i \sigma}{r-2 M}\right)} \int \frac{\mathrm{e}^{\left(-\frac{r i \sigma}{-r+2 M}\right)}(r N(r)-r L(r))}{-r+2 M} \mathrm{~d} r .
$$

After the above transformation, we note that $\mathrm{e}^{i \sigma t}$ now has the form $\mathrm{e}^{i \sigma u+i \sigma F(r)}$. We also note that from the transformed metric there is a $\operatorname{d} r d \theta$ term that needs to be removed. We remove this term by transforming $\theta$ to $\psi$ by performing the following transformation

$$
\theta \rightarrow \psi=\theta-\varepsilon \alpha(u, r, \psi)
$$

where $\alpha(u, r, \psi)$ is a function that needs to be determined. We then differentiate Equation (29) to get

$$
\mathrm{d} \theta=\mathrm{d} \psi+\varepsilon \alpha(u, r, \psi)_{, u} \mathrm{~d} u+\varepsilon \alpha(u, r, \psi)_{, r} \mathrm{~d} r+\varepsilon \alpha(u, r, \psi)_{, \psi} \mathrm{d} \psi
$$

We substitute Equation (30) into the transformed metric and apply the condition that the coefficient of $\mathrm{d} r \mathrm{~d} \psi$ must be zero to 1 st order in $\varepsilon$. We then work out the complete transformed metric up to 1st order in $\varepsilon$ and transform $\sin ^{2} \theta$ as follows

$$
\sin ^{2} \theta \rightarrow \sin ^{2}(\psi-\varepsilon \alpha(u, r, \psi))=(\sin \psi-\varepsilon \alpha(u, r, \psi) \cos \psi)^{2}=\sin ^{2} \psi(1-2 \varepsilon \alpha(u, r, \psi) \cot \psi)
$$

We found $\alpha(u, r, \psi)$ to be

$$
\alpha(u, r, \psi)=-3 \sin \psi \cos \psi \mathrm{e}^{\mathrm{i} \sigma u} I_{f}(r),
$$

where

$$
I_{f}(r)=\int \frac{\mathrm{e}^{\mathrm{i} \sigma F(r)} f(r)}{r^{2}} \mathrm{~d} r
$$

Finally, we transform $r$ to a new $r^{\prime}$ by performing the following transformation

$$
r \rightarrow r^{\prime}=r+\varepsilon \Omega(u, r, \psi)
$$

were $\Omega(u, r, \psi)$ is a function that needs to be determined. Equation (34) satisfy the following condition

$$
r^{\prime 4} \sin ^{2} \psi=g_{22} \cdot g_{33} .
$$

We use Equation (35) to find $\Omega(u, r, \psi)$, and it was found to be

$$
\Omega(u, r, \psi)=-\frac{1}{4} \mathrm{e}^{i \sigma u}\left(3 \cos ^{2} \psi-1\right) r\left[3 I_{f}(r)+\mathrm{e}^{i \sigma F(r)}[3 V(r)-T(r)]\right] .
$$

\subsubsection{The Transformed Metric}

After the above transformation, we found the transformed metric to be given by

$$
\begin{aligned}
g_{00}= & -\frac{1}{r^{2}}\left[-2 M r-2 \varepsilon\left[\frac{\partial}{\partial u} \Omega(u, r, \psi)\right] r^{2}-2 M \delta \Omega(u, r, \psi)+r^{2}\right. \\
& +2 \mathrm{e}^{(i \sigma F(r)+i \sigma u)} \varepsilon f(r) i \sigma P_{2}(\psi) r^{2}-4 \mathrm{e}^{(i \sigma F(r)+i \sigma u)} \varepsilon f(r) i \sigma P_{2}(\psi) M r \\
& \left.-4 \mathrm{e}^{(i \sigma F(r)+i \sigma u)} \varepsilon P_{2}(\psi) N(r) M r+2 \mathrm{e}^{(i \sigma F(r)+i \sigma u)} \varepsilon P_{2}(\psi) N(r) r^{2}\right], \\
g_{01}= & g_{10}=-1+\varepsilon\left[\frac{\partial}{\partial r} \Omega(u, r, \psi)\right]-2 \mathrm{e}^{(i \sigma F(r)+i \sigma u)} \varepsilon P_{2}(\psi) N(r) \\
& -2 \mathrm{e}^{(i \sigma F(r)+i \sigma u)} \varepsilon f(r) i \sigma P_{2}(\psi),
\end{aligned}
$$




$$
\begin{aligned}
g_{02}= & g_{20}=\frac{\varepsilon}{r}\left[\left[\frac{\partial}{\partial \psi} \Omega(u, r, \psi)\right] r+2 \mathrm{e}^{(i \sigma F(r)+i \sigma u)} f(r)\left[\frac{\partial}{\partial \psi} P_{2}(\psi)\right] M\right. \\
& \left.+\left[\frac{\partial}{\partial u} \Omega(u, r, \psi)\right] r^{3}-\mathrm{e}^{(i \sigma F(r)+i \sigma u)} f(r)\left[\frac{\partial}{\partial \psi} P_{2}(\psi)\right] r\right], \\
g_{22}= & {\left[-2 \varepsilon \Omega(u, r, \psi)+r-12 \mathrm{e}^{(i \sigma F(r)+i \sigma u)} r \varepsilon V(r) \cos ^{2} \psi+2 \mathrm{e}^{(i \sigma F(r)+i \sigma u)} r \varepsilon P_{2}(\psi) T(r)\right.} \\
& 6 \mathrm{e}^{(i \sigma F(r)+i \sigma u)} r \varepsilon V(r)+2 r \varepsilon\left[\frac{\partial}{\partial \psi} \Omega(u, r, \psi)\right], \\
g_{33}= & -r\left[2 \varepsilon \Omega(u, r, \psi)+r \cos ^{2} \psi-r+2 \sin \psi r \varepsilon \alpha \cos ^{2} \psi-2 \cos ^{2} \psi \varepsilon \Omega(u, r, \psi)\right. \\
& +6 \mathrm{e}^{(i \sigma F(r)+i \sigma u)} r \varepsilon V(r) \cos ^{2} \psi-6 \mathrm{e}^{(i \sigma F(r)+i \sigma u)} r \varepsilon \cos ^{4} \psi V(r) \\
& \left.-2 \mathrm{e}^{(i \sigma F(r)+i \sigma u)} r \varepsilon P_{2}(\psi) T(r)+2 \mathrm{e}^{(i \sigma F(r)+i \sigma u)} r \varepsilon P_{2}(\psi) T(r) \cos ^{2} \psi\right] .
\end{aligned}
$$

which simplifies to

$$
\begin{aligned}
g_{00}= & -1+\frac{2 M}{r}+2 \varepsilon \mathrm{e}^{i \sigma u} P_{2}(\psi) \mathrm{e}^{i \sigma F(r)}\left[\frac{2 M}{r}-1\right][f(r) i \sigma+N(r)] \\
& +\frac{1}{r}\left[2 \varepsilon\left[\frac{\partial}{\partial u} \Omega(u, r, \psi)\right] r+\frac{2 M}{r} \varepsilon \Omega(u, r, \psi)\right], \\
g_{01}= & g_{10}=-1-\left[\varepsilon\left[\frac{\partial}{\partial r} \Omega(u, r, \psi)\right]+2 \mathrm{e}^{i \sigma u} \varepsilon P_{2}(\psi) \mathrm{e}^{i \sigma F(r)}[f(r) i \sigma+N(r)]\right], \\
g_{02}= & g_{20}=-r^{2}\left[-\varepsilon\left[\frac{\partial}{\partial \psi} \Omega(u, r, \psi)\right] \frac{1}{r^{2}}-2 \varepsilon \mathrm{e}^{i \sigma u}\left[\frac{\partial}{\partial} P_{2}(\psi)\right] \mathrm{e}^{i \sigma F(r)} f(r) \frac{M}{r^{3}}\right. \\
& \left.-\varepsilon\left[\frac{\partial}{\partial u} \alpha(u, r, \psi)\right]+\varepsilon \mathrm{e}^{i \sigma u}\left[\frac{\partial}{\partial \psi} P_{2}(\psi)\right] \mathrm{e}^{i \sigma F(r)} f(r) \frac{1}{r^{2}}\right], \\
g_{22}= & r^{2}\left[1+\left[-\frac{2 \varepsilon}{r} \Omega(u, r, \psi)+2 \mathrm{e}^{i \sigma u} \varepsilon P_{2}(\psi) \mathrm{e}^{i \sigma F(r)} T(r)\right.\right. \\
& \left.+6 \mathrm{e}^{i \sigma u} \varepsilon\left[1-2 \cos { }^{2} \psi\right] \mathrm{e}^{i \sigma F(r)} V(r)+2 \varepsilon\left[\frac{\partial}{\partial \psi} \alpha(u, r, \psi)\right]\right], \\
& \left.\left.-6 \varepsilon \cos ^{2} \psi \mathrm{e}^{i \sigma u} I_{f}(r)\right]\right] \sin ^{2} \psi, \\
g_{33}= & r^{2}\left[1-\left[\frac{2 \varepsilon}{r} \Omega(u, r, \psi)+6 \mathrm{e}^{i \sigma u} \cos ^{2} \psi \varepsilon \mathrm{e}^{i \sigma F(r)} V(r)-2 \mathrm{e}^{i \sigma u} \varepsilon P_{2}(\psi) \mathrm{e}^{i \sigma F(r)} T(r)\right.\right. \\
& {[(r)]] }
\end{aligned}
$$

$F(r), f(r), \alpha(r), \Omega(u, r, \psi)$ are functions given by Equations (27), (28), (32), and (36) respectively.

\subsubsection{The Comparison}

By comparing the transformed even-parity metric perturbations with the linearized Bondi-Sachs metric (see

Section 2) and noticing that $g_{11}=g_{12}=g_{13}=g_{21}=g_{31}=g_{23}=g_{32}=g_{03}=g_{30}=0$, we found that $\beta, U, J, w$ 
are given by

$$
\begin{gathered}
w=-\left[2 \varepsilon\left[\frac{\partial}{\partial u} \Omega(u, r, \psi)\right] r+\frac{2 M}{r} \Omega(u, r, \psi)\right], \\
\beta=\left[\varepsilon\left[\frac{\partial}{\partial r} \Omega(u, r, \psi)\right]+2 \mathrm{e}^{i \sigma u} \varepsilon P_{2}(\psi) \mathrm{e}^{i \sigma F(r)}[f(r) i \sigma+N(r)]\right], \\
U=2\left[-\varepsilon\left[\frac{\partial}{\partial} \Omega(u, r, \psi)\right] \frac{1}{r^{2}}-2 \varepsilon \mathrm{e}^{i \sigma u}\left[\frac{\partial}{\partial \psi} P_{2}(\psi)\right] \mathrm{e}^{i \sigma F(r)} f(r) \frac{M}{r^{3}}\right. \\
\left.-\varepsilon\left[\frac{\partial}{\partial u} \alpha(u, r, \psi)\right]+\varepsilon \mathrm{e}^{i \sigma u}\left[\frac{\partial}{\partial \psi} P_{2}(\psi)\right] \mathrm{e}^{i \sigma F(r)} f(r) \frac{1}{r^{2}}\right], \\
J=-\frac{2 \varepsilon}{r} \Omega(u, r, \psi)+2 \mathrm{e}^{i \sigma u} \varepsilon P_{2}(\psi) \mathrm{e}^{i \sigma F(r)} T(r)+6 \mathrm{e}^{i \sigma u} \varepsilon\left[1-2 \cos ^{2} \psi\right] \mathrm{e}^{i \sigma F(r)} V(r)+2 \varepsilon\left[\frac{\partial}{\partial \psi} \alpha(u, r, \psi)\right],
\end{gathered}
$$

or

$$
J=\frac{2 \varepsilon}{r} \Omega(u, r, \psi)+6 \mathrm{e}^{i \sigma u} \cos ^{2} \psi \varepsilon \mathrm{e}^{i \sigma F(r)} V(r)-2 \mathrm{e}^{\mathrm{i} \sigma u} \varepsilon P_{2}(\psi) \mathrm{e}^{i \sigma F(r)} T(r)-6 \varepsilon \cos ^{2} \psi \mathrm{e}^{\mathrm{i} \sigma u} I_{f}(r) .
$$

By substituting functions (27), (28), (32), and (36) into Equations (47), (48), (49), and (50), $\omega, \beta, U$, and $J$ simplify to

$$
w=w_{0}(r) \varepsilon \mathrm{e}^{i \sigma u} Y_{20}(\theta)
$$

where

$$
\begin{gathered}
w_{0}(r)=\left(\frac{4 \pi^{\frac{1}{2}}}{5^{\frac{1}{2}}}\right) \frac{1}{2} r\left[i \sigma r+\frac{M}{r}\right]\left[3 I_{f}(r)+\mathrm{e}^{i \sigma F(r)}[3 V(r)-T(r)]\right], \\
\beta=\beta_{0}(r) \varepsilon \mathrm{e}^{i \sigma u} Y_{20}(\theta),
\end{gathered}
$$

where

$$
\begin{gathered}
\beta_{0}(r)=-\left(\frac{4 \pi^{\frac{1}{2}}}{5^{\frac{1}{2}}}\right)\left[\frac { 1 } { 4 } \left\{3\left[I_{f}(r)+r I_{f}^{\prime}(r)\right]+\mathrm{e}^{i \sigma F(r)}\left[3\left[\left(1-r i \sigma F^{\prime}(r)\right) V(r)+r V^{\prime}(r)\right]\right.\right.\right. \\
\left.\left.\left.-\left[(1-r i \sigma F(r)) T(r)+r T^{\prime}(r)\right]\right]\right\}+\mathrm{e}^{i \sigma F(r)}[f(r) i \sigma+N(r)]\right] \\
U=\varepsilon \mathrm{e}^{i \sigma u}{ }_{1} Y_{20} U_{0}(r),
\end{gathered}
$$

where

$$
\begin{gathered}
U_{0}(r)=\frac{1}{2}\left[-\frac{1}{2 r}\left[3 I_{f}(r)+\mathrm{e}^{i \sigma F(r)}[3 V(r)-T(r)]\right]+I_{f}(r)+\frac{\mathrm{e}^{i \sigma F(r)}}{r^{2}}[2 M-1]\right] \\
J=\varepsilon \mathrm{e}^{i \sigma u}{ }_{2} Y_{20} J_{0}(r)
\end{gathered}
$$

where 


$$
\begin{aligned}
J_{0}(r)= & \left(\frac{4 \pi^{\frac{1}{2}}}{5^{\frac{1}{2}}}\right) \frac{1}{2 \sqrt{6}}\left\{-\frac{1}{2}\left[\frac{1}{2}\left[3 I_{f}(r)+\mathrm{e}^{i \sigma F(r)}[3 V(r)-T(r)]\right]+\mathrm{e}^{i \sigma F(r)} T(r)\right]\right. \\
& \left.+2\left[\mathrm{e}^{i \sigma F(r)} V(r)+I_{f}(r)\right]\right\} .
\end{aligned}
$$

We have used the trigonometric identities:

$$
\cos a \cos b=\frac{1}{2}[\cos (a-b)-\cos (a+b)] \text { and } \sin ^{2} \theta+\cos ^{2} \theta
$$

to simplify $\frac{3}{2} \cos ^{2} \psi-\frac{1}{2}$ to $\frac{1}{4}[3(\cos 2 \psi-1)]$ in Equation (50).

\subsubsection{Interpreting the Complex Quantities}

The expressions for $\beta, w, J$ and $U$ obtained above involve the complex quantity $i$, but not $j$. Thus, here, the interpretation is straightforward: in all cases $\beta, w, J$ and $U$ mean the real part of the given expression.

\subsection{Odd-Parity Metric Perturbations}

\subsubsection{The Transformation Procedure}

From Equation (23) we have

$$
\begin{aligned}
\mathrm{d} s^{2}= & -\left(1-\frac{2 M}{r}\right) \mathrm{d} t^{2}+\left(1-\frac{2 M}{r}\right)^{-1} \mathrm{~d} r^{2}+r^{2} \mathrm{~d} \theta^{2} \\
& +r^{2} \sin ^{2} \theta\left(\mathrm{d} \phi-\omega(r, \theta, u) \mathrm{d} t-q_{2}(r, \theta, t) \mathrm{d} r-q_{3}(r, \theta, t) \mathrm{d} \theta\right)^{2},
\end{aligned}
$$

since $\omega(r, \theta, t), q_{2}(r, \theta, t)$ and $q_{3}(r, \theta, t)$ are very small, we then have

$$
\begin{aligned}
& \omega(r, \theta, t)=\varepsilon \omega(r) 3 \cos \theta \mathrm{e}^{i \sigma t}, \\
& q_{2}(r, \theta, t)=\varepsilon q_{2}(r) 3 \cos \theta \mathrm{e}^{i \sigma t}, \\
& q_{3}(r, \theta, t)=\varepsilon q_{3}(r) 3 \sin \theta \mathrm{e}^{i \sigma t} .
\end{aligned}
$$

We start by transforming $t$ to $u$ by the following transformation

$$
u=t-F(r) \text {, }
$$

where $F(r)$ is a function that needs to be determined. Differentiating this transformation we obtain

$$
\mathrm{d} t=\mathrm{d} u+F^{\prime}(r) \mathrm{d} r .
$$

By substituting Equation (66) into Equation (61) and choosing the function $F(r)$ such that the transformed metric after the substitution of Equation (66) has the coefficient of $d r^{2}$ zero to the zeroth order in $\varepsilon$ and we note that $\mathrm{e}^{i \sigma t}$ now has the form $\mathrm{e}^{i \sigma(u+F(r))}$. We found the transformed metric to be

$$
\begin{aligned}
\mathrm{d} s^{2}= & -\left(1-\frac{2 M}{r}\right) \mathrm{d} u^{2}-2\left(1-\frac{2 M}{r}\right)\left(\frac{\partial}{\partial_{r}} F(r)\right) \mathrm{d} u \mathrm{~d} r+r^{2} \mathrm{~d} \theta^{2} \\
& +r^{2} \sin \theta^{2}\left(\mathrm{~d} \phi-\varepsilon \omega(r) 3 \cos \theta \mathrm{e}^{i \sigma(u+F(r))} \mathrm{d} u-\left(\varepsilon \omega(r) 3 \cos \theta \mathrm{e}^{i \sigma(u+F(r))} \frac{\partial}{\partial_{r}} F(r)\right.\right. \\
& \left.\left.+\varepsilon q_{2}(r) 3 \cos \theta \mathrm{e}^{i \sigma(u+F(r))}\right) \mathrm{d} r-\varepsilon q_{3}(r) 3 \sin \theta \mathrm{e}^{i \sigma(u+F(r))} \mathrm{d} \theta\right)^{2},
\end{aligned}
$$


where a function $F(r)$ was found to be

$$
F(r)=r+2 M \ln (r-2 M) .
$$

We then transform $\phi$ to $\psi$ by performing the following transformation

$$
\psi=\phi+g(r, \theta, u)
$$

where $g(r, \theta, u)$ is a function that needs to be determined. Differentiating this transformation we obtain

$$
\mathrm{d} \phi=\mathrm{d} \psi-\left[\frac{\partial}{\partial r} g(r, \theta, u)\right] \mathrm{d} r-\left[\frac{\partial}{\partial \theta} g(r, \theta, u)\right] \mathrm{d} \theta-\left[\frac{\partial}{\partial u} g(r, \theta, u)\right] \mathrm{d} u .
$$

Then by substituting Equation (70) into Equation (67) and choosing $g(r, \theta, u)$ such that the transformed metric after the substitution has the coefficient of $d r^{2}$ zero we get

$$
\begin{aligned}
\mathrm{d} s^{2}= & -\left(1-\frac{2 M}{r}\right) \mathrm{d} u^{2}-2 \mathrm{~d} u \mathrm{~d} r+r^{2} \mathrm{~d} \theta^{2}+r^{2} \sin ^{2} \theta\left[\mathrm{d} \psi-\left[\frac{\partial}{\partial \theta} g(r, \theta, u)\right] \mathrm{d} \theta\right. \\
& \left.-\left[\frac{\partial}{\partial u} g(r, \theta, u)\right] \mathrm{d} u-\varepsilon \omega(r) 3 \cos \theta \mathrm{e}^{i \sigma(u+F(r))} \mathrm{d} u-\varepsilon q_{3}(r) 3 \sin \theta \mathrm{e}^{i \sigma(u+F(r))} \mathrm{d} \theta\right]^{2},
\end{aligned}
$$

where a function $g(r, \theta, u)$ was found to be

$$
g(r, \theta, u)=-3 \varepsilon \cos \theta \mathrm{e}^{\mathrm{i} \sigma u}\left[I_{\omega}(r)+I_{q_{2}}(r)\right]
$$

where

$$
I_{\omega}(r)=\int \frac{\mathrm{e}^{i \sigma F(r)}[-\omega(r) r]}{-r+2 M} \mathrm{~d} r \text { and } I_{q_{2}}(r)=\int \mathrm{e}^{\mathrm{i} \sigma F(r)} q_{2}(r) \mathrm{d} r
$$

\subsubsection{The Transformed Metric}

After the above transformation procedure, we found the transformed metric to be

$$
\begin{aligned}
g_{00}= & -\frac{1}{r}\left[r-2 M-r^{3}\left[\frac{\partial}{\partial u} g(r, \theta, u)\right]^{2}+r^{3}\left[\frac{\partial}{\partial u} g(r, \theta, u)\right]^{2} \cos ^{2} \theta\right. \\
& -6 r^{3}\left[\frac{\partial}{\partial u} g(r, \theta, u)\right] \varepsilon \omega(r) \cos \theta \mathrm{e}^{i \sigma(u+F(r))} \\
& \left.+6 r^{3}\left[\frac{\partial}{\partial u} g(r, \theta, u)\right] \varepsilon \omega(r) \cos ^{3} \theta \mathrm{e}^{i \sigma(u+F(r))}\right], \\
g_{01}= & -1, \\
g_{02}= & -r^{2}(\cos \theta-1)(\cos \theta+1)\left[3\left[\frac{\partial}{\partial \theta} g(r, \theta, u)\right] \varepsilon \omega(r) \cos \theta \mathrm{e}^{i \sigma(u+F(r))}\right. \\
& \left.+\left[\frac{\partial}{\partial \theta} g(r, \theta, u)\right]\left[\frac{\partial}{\partial u} g(r, \theta, u)\right]+3 \sin \theta\left[\frac{\partial}{\partial u} g(r, \theta, u)\right] \times \varepsilon q_{3}(r) \mathrm{e}^{i \sigma(u+F(r))}\right], \\
g_{03}= & r^{2}(\cos \theta-1)(\cos \theta+1)\left[3 \varepsilon \omega(r) \cos \theta \mathrm{e}^{i \sigma(u+F(r))}+\left[\frac{\partial}{\partial u} g(r, \theta, u)\right]\right] \\
g_{23}= & r^{2}(\cos \theta-1)(\cos \theta+1)\left[\left[\frac{\partial}{\partial \theta} g(r, \theta, u)\right]+3 \varepsilon \sin \theta q_{3}(r) \mathrm{e}^{i \sigma(u+F(r))}\right]
\end{aligned}
$$




$$
\begin{aligned}
g_{22}= & -r^{2}\left[-1-\left[\frac{\partial}{\partial \theta} g(r, \theta, u)\right]^{2}+\left[\frac{\partial}{\partial \theta} g(r, \theta, u)\right]^{2} \cos ^{2} \theta\right. \\
& -6 \sin \theta\left[\frac{\partial}{\partial \theta} g(r, \theta, u)\right] \varepsilon q_{3}(r) \mathrm{e}^{i \sigma(u+F(r))} \\
& \left.+6 \sin \theta\left[\frac{\partial}{\partial \theta} g(r, \theta, u)\right] \varepsilon q_{3}(r) \mathrm{e}^{i \sigma(u+F(r))} \cos ^{2} \theta\right], \\
g_{33}= & -r^{2}(\cos \theta-1)(\cos \theta+1) .
\end{aligned}
$$

Substituting Equation (72) in the above metric components, they simplify to

$$
\begin{aligned}
& g_{00}=-1+\frac{2 M}{r}, \\
& g_{01}=-1, \\
& g_{02}=0, \\
& g_{03}=-3 r^{2} \sin ^{2} \theta \varepsilon \cos \theta \operatorname{Re}\left\{\mathrm{e}^{i \sigma u}\left[\omega(r) \mathrm{e}^{i \sigma F(r)}-i \sigma\left(I_{\omega}(r)+I_{q_{2}}(r)\right)\right]\right\}, \\
& g_{23}=-3 r^{2} \sin ^{3} \theta \varepsilon \operatorname{Re}\left\{\mathrm{e}^{i \sigma u}\left[I_{\omega}(r)+I_{q_{2}}(r)+q_{3}(r) \mathrm{e}^{i \sigma F(r)}\right]\right\}, \\
& g_{22}=r^{2}, \\
& g_{33}=r^{2} \sin ^{2} \theta .
\end{aligned}
$$

\subsubsection{The Comparison}

By comparing the transformed odd-parity metric perturbations with the linearized Bondi-Sachs metric (see Section 2) we found that $\beta, U, J, w \beta_{0}, U_{0}, J_{0}$, and $w_{0}$ for the transformed odd-parity metric perturbations are given by

$$
\begin{gathered}
\beta=0, \text { and hence } \beta_{0}(r)=0 . \\
w=0, \text { and hence } w_{0}=0 .
\end{gathered}
$$

From Equation (17) with $a=0$ we have

$$
J=\frac{j b}{\sin \theta},
$$

From Equation (16) with $g_{02}=0$ we have

$$
U=-\frac{1}{r^{2}} \frac{j g_{03}}{\sin \theta},
$$

\subsubsection{Interpreting the Complex Quantities}

The expressions for $J$ and $U$ obtained above involve both complex quantities $i$ and $j$. Taking the real part with respect to $i$ leads to

$$
J=-j \varepsilon_{2} Y_{20}\left(\frac{4 \pi^{\frac{1}{2}}}{5^{\frac{1}{2}}}\right) \operatorname{Re}\left\{\mathrm{e}^{i \sigma u}\left[I_{\omega}(r)+I_{q_{2}}(r)+q_{3}(r) \mathrm{e}^{i \sigma F(r)}\right]\right\}
$$

and 


$$
U=j \varepsilon_{1} Y_{20}\left(\frac{4 \pi^{\frac{1}{2}}}{5^{\frac{1}{2}}}\right) \operatorname{Re}\left\{\mathrm{e}^{i \sigma u}\left[\omega(r) \mathrm{e}^{i \sigma F(r)}-i \sigma\left(I_{\omega}(r)+I_{q_{2}}(r)\right)\right]\right\}
$$

Thus, both $U$ and $J$ are pure imaginary quantities.

\section{Discussion}

The transformation of linear perturbations of a Schwarzschild black hole to Bondi-Sachs is complete. The transformation of even-parity perturbations was much more involved than that of odd-parity perturbations. The end results of the transformation processes for both even and odd-parity perturbations were very different, for example, in the case of odd-parity perturbations, $w$ and $\beta$ were found to be zero and $J$ and $U$ were found to be purely imaginary and that was not the case for even-parity perturbations were $w, \beta, J$ and $U$ were found to be real and complicated functions.

All unknown functions; $f(r), F(r), \alpha(u, r, \psi), \Omega(u, r, \psi)$ and $g(r, \theta, u)$ for both even and odd-parity perturbations, were found and verified to be correct by substituting them into the transformed even and odd-parity perturbations, thereby simplifying the transformed perturbations to a point where we were able to find $J, U, w$, and $\beta$. We then wrote $J, U, w$, and $\beta$ as spherical harmonics $\left({ }_{2} Y_{20},{ }_{1} Y_{20}, Y_{20}\right.$ and $Y_{20}$ respectively) times some functions ( $J_{0}, U_{0}, w_{0}$, and $\beta_{0}$ respectively) times the time dependency factor $\mathrm{e}^{\mathrm{i} \sigma u}$. Also, for the fact that we were able to extract $Y_{20},{ }_{1} Y_{20},{ }_{2} Y_{20}$ and $J_{0}, U_{0}, w_{0}, \beta_{0}$ from the transformed odd and even-parity perturbations, meant that the transformation processes were carried out correctly and that all the unknown functions $f(r), F(r), \alpha(u, r, \psi), \Omega(u, r, \psi)$ and $g(r, \theta, u)$ were correctly determined.

\section{Conclusion}

It appears that the transformation of second order perturbations of a Schwarzschild black hole to Bondi-Sachs form will be extremely difficult to do. In the future, the extension of the work of this paper to a stationary charged (Reissener-Nordström) black hole will be very exciting and hopefully attainable. Similarly, the transformation of linear perturbations(gravitational) of a Kerr black hole will be very exciting to do, but the transformation of its standard metric to Bondi-Sachs form has been obtained only very recently [18] and is not in an explicit analytic form yet. In addition, if we extend the work of this dissertation to Kerr-Newman black hole, we will find it difficult to transform linear perturbations because even and odd-parity perturbations have not yet been decoupled and this is still a challenge to us.

\section{Acknowledgements}

ASK and NTB would like to thank the University of South African, Rhodes University, and National Research Foundation of South Africa for the financial support.

\section{References}

[1] Seidel, E. (1998) Numerical Relativity: Towards Simulations of 3D Black Hole Coalescence. In: Iyer, B. and Bhawal, B., Eds., On the Black Hole Trail, Kluwer, Report-No.: AEI-066.

[2] Seidel, E. (1998) Plenary Talk Given at GR15. Proceedings of the GR-15 Conference, Poona, Report-No.: AEI-071.

[3] Arnowitt, R., Deser, S. and Misner, C.W. (1962) Gravitation: An Introduction to Current Research. Wiley, New York.

[4] York, J.W. (1979) Sources of Gravitational Radiation. Cambridge University Press, Seatle.

[5] Bondi, H., van der Burg, M.J.G. and Metzner, A.W.K. (1962) Proceedings of the Royal Society London A, 269, 21-52. http://dx.doi.org/10.1098/rspa.1962.0161

[6] Sachs, R.K. (1962) Proceedings of the Royal Society London A, 270, 103-126. http://dx.doi.org/10.1098/rspa.1962.0206

[7] Bartnik, R. (1997) Classical and Quantum Gravity, 14, 2185-2194. http://dx.doi.org/10.1088/0264-9381/14/8/017

[8] Bishop, N.T., Gomez, R., Husa, S., Lehner, L. and Winicour, J. (2003) Physical Review D, 68, Article ID: 084015. http://dx.doi.org/10.1103/PhysRevD.68.084015

[9] Bishop, N.T. and Deshingkar, S.S. (2003) Physical Review D, 68, Article ID: 024031. 
http://dx.doi.org/10.1103/PhysRevD.68.024031

[10] d'Inverno, R.A., Dubal, M.R. and Sarkies, E.A. (2001) Classical and Quantum Gravity, 17, 3157-3170. http://dx.doi.org/10.1088/0264-9381/17/16/305

[11] Gómez, R. (2001) Physical Review D, 64, Article ID: 024007. http://dx.doi.org/10.1103/PhysRevD.64.024007

[12] Siebel, F., Font, J.A., Muller, E. and Papadopoulos, P. (2003) Physical Review D, 67, Article ID: 124018 http://dx.doi.org/10.1103/PhysRevD.67.124018

[13] Sachs, R.K. (1963) Physical Review Letters, 150, 66.

[14] Bishop, N.T., Gómez, R., Lehner, L., Maharaj, M. and Winicour, J. (1997) Physical Review D, 56, 6298. http://dx.doi.org/10.1103/PhysRevD.56.6298

[15] Gómez, R., Lehner, L., Papadopoulos, P. and Winicour, J. (1997) Classical and Quantum Gravity, 14, 977-990. http://dx.doi.org/10.1088/0264-9381/14/4/013

[16] Bishop, N.T. (2005) Classical and Quantum Gravity, 22, 2393-2406. http://dx.doi.org/10.1088/0264-9381/22/12/006

[17] Chandrasekhar, S. and Friedmann, J.L. (1972) Astrophysical Journal, 175, 379. http://dx.doi.org/10.1086/151566

[18] Venter, L.R. and Bishop, N.T. (2006) Physical Review D, 73, Article ID: 084023.

http://dx.doi.org/10.1103/PhysRevD.73.084023 
Scientific Research Publishing (SCIRP) is one of the largest Open Access journal publishers. It is currently publishing more than 200 open access, online, peer-reviewed journals covering a wide range of academic disciplines. SCIRP serves the worldwide academic communities and contributes to the progress and application of science with its publication.

Other selected journals from SCIRP are listed as below. Submit your manuscript to us via either submit@scirp.org or Online Submission Portal.
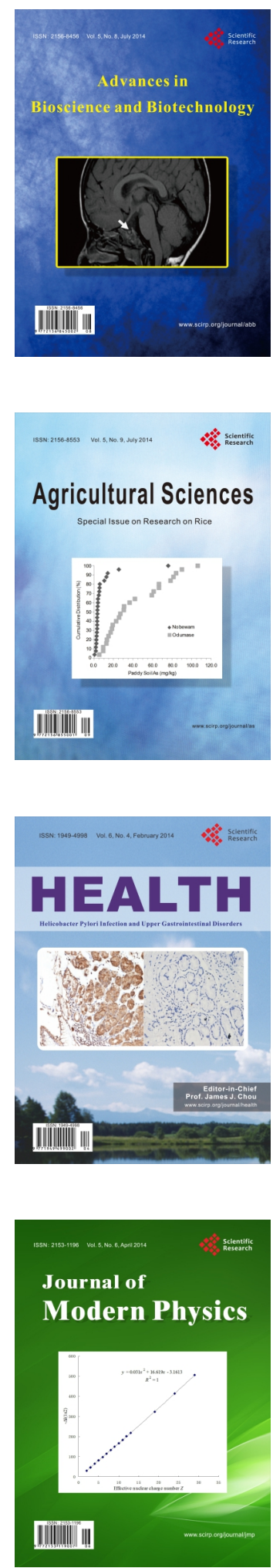
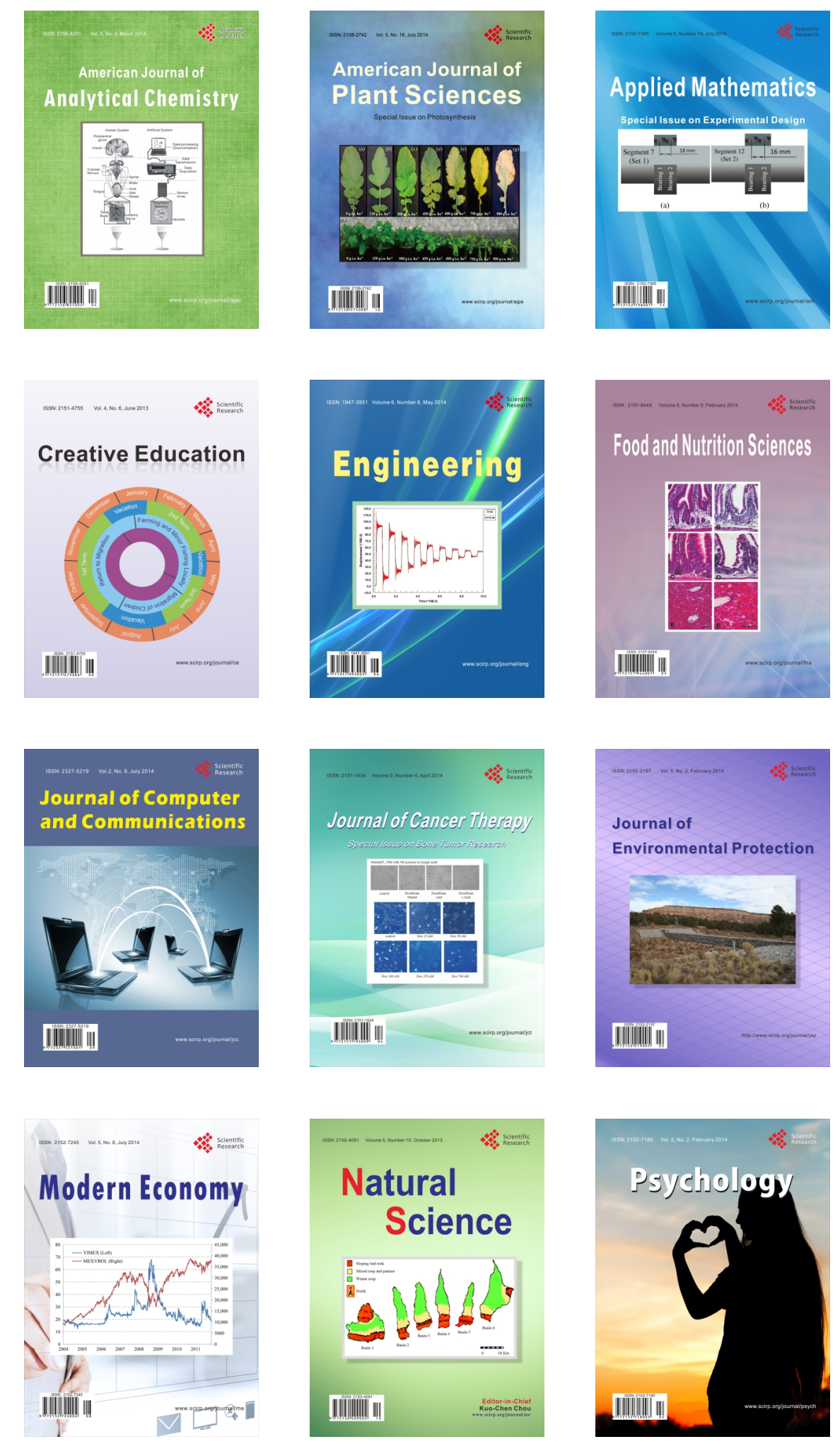